\title{
The use of the isometry of function spaces with different numbers of variables in the theory of approximation of functions
}

\begin{abstract}
Bushev D.M. ${ }^{1}$, Abdullayev F.G. ${ }^{2,3, \bowtie}$, Kal'chuk I.V. ${ }^{1}$, Imashkyzy M. ${ }^{3}$
In the work, we found integral representations for function spaces that are isometric to spaces of entire functions of exponential type, which are necessary for giving examples of equality of approximation characteristics in function spaces isometric to spaces of non-periodic functions. Sufficient conditions are obtained for the nonnegativity of the delta-like kernels used to construct isometric function spaces with various numbers of variables.

Key words and phrases: delta-like kernel, isometry, space of convolutions, approximative characteristic.
\end{abstract}

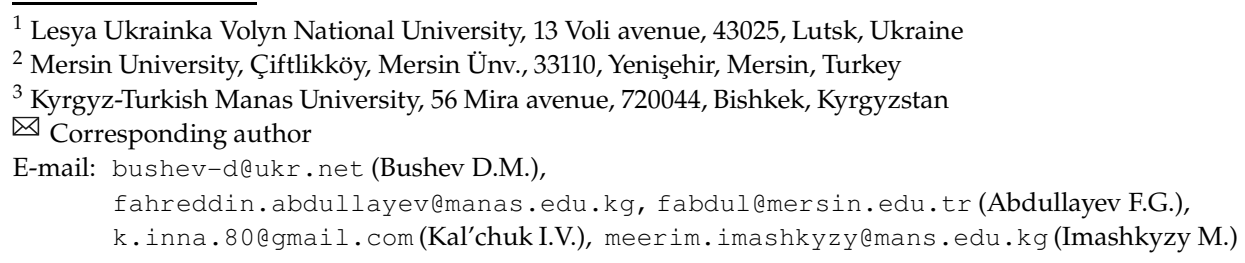

\section{Introduction}

In the paper [4], there were constructed the spaces of real $n+k$ variable functions that are isomeric to functions of real variables, defined on the $n$-dimensional Euclidean space. In view of the fact, that isometry of functional spaces with different numbers of variables is a rare phenomenon that was previously known only for the spaces of complex-valued functions (see [4, p. 1027]), it is expedient to consider its application.

In [5], there were found the subspaces of solutions of Laplace and thermal conductivity equations that are isometric to the spaces of real functions of one variable. In the paper [1], the authors got the subspaces of solutions of systems of Laplace and thermal conductivity equations that are isometric to spaces of real functions.

To construct subspaces of solutions of differential equations and their systems that are isometric to the spaces of real functions, it was necessary to establish the conditions for convergence of a convolution of the function with its delta-like kernel. This was made in the paper [6].

In $[7,8]$, the main approximative characteristics were defined, and the examples of their equality for isometric mappings of the spaces of real $n+m$ variable functions, on spaces of real $2 \pi$-periodic in each of $n$ variables functions. Also, there are some examples of application of isometry in the theory of approximation of functions.

$\mathrm{y} \triangle \mathrm{K} 517.5$

2020 Mathematics Subject Classification:30C30, 30E10, 30C70.

This work is supported by Kyrgyz-Turkish Manas University [KTMU-BAP.2019.FBE.03]. 
In this paper, we extend the results of $[7,8]$ to isometric mappings in the spaces of nonperiodic functions, find integral representations for function spaces that are isometric to spaces of entire functions of exponential type, and establish sufficient conditions for operators to map convolutions of spaces of function given on $n$-dimensional Euclidean space, on the spaces of entire functions of exponential type.

Let us denote with $\widetilde{C}, \widetilde{L}_{\infty}, \widetilde{L}_{p}$ the spaces of real functions, defined on the interval $[-\pi, \pi)$, that are $2 \pi$-periodic in each variable $x$ and, respectively, essentially bounded and measurable functions with the norms

$$
\|f\|_{\widetilde{C}}=\sup _{x \in[-\pi, \pi)}|f(x)|, \quad\|f\|_{\widetilde{\infty}^{n}}=\sup _{x \in[-\pi, \pi)} \operatorname{vrai}|f(x)|, \quad\|f\|_{\widetilde{p}}=\left(\int_{-\pi}^{\pi}|f(x)|^{p} d x\right)^{1 / p}
$$

where $1 \leq p<\infty$.

The inequality $\bar{y}=\left(y_{1}, \ldots, y_{n}\right) \geq \overline{0}=(0, \ldots, 0)$ means that the coordinates of vector $\bar{y}$ are non-negative, and $\bar{y}>\overline{0}$ means that at least one of them is positive,

$$
\begin{aligned}
\Pi_{n, m}^{+}=\{(x, y)= & \left.(\bar{x}, \bar{y})=\left(x_{1}, \ldots, x_{n}, y_{1}, \ldots, y_{m}\right) \in E^{n+m}:(\bar{y}>\overline{0})\right\}, \\
& \bar{\Pi}_{n, m}^{+}=\left\{(x, y) \in E^{n+m}:(\bar{y} \geq \overline{0})\right\}
\end{aligned}
$$

are the subspaces of real $(n+m)$-dimensional Euclidean space $E^{n+m}, \widetilde{X}$ is one of the spaces $\widetilde{C}$, $\widetilde{L}_{\infty}, \widetilde{L}_{p}, \widetilde{X} M_{m}$, and $\widetilde{X} \bar{M}_{m}$ are the spaces of real functions $f(x, y)=f(x, \bar{y})=f\left(x, y_{1}, \ldots, y_{m}\right)$, defined, respectively, on the sets $\Pi_{1, m}^{+}$and $\bar{\Pi}_{1, m}^{+}$with the norms

$$
\|f\|_{\widetilde{X} M_{m}}=\sup _{\bar{y}>\overline{0}}\|f(x, \bar{y})\|_{\widetilde{X}^{\prime}} \quad\|f\|_{\widetilde{X} \bar{M}_{m}}=\sup _{\bar{y} \geq \overline{0}}\|f(x, \bar{y})\|_{\widetilde{X}}
$$

Let further $Q_{\delta}=[-\delta, \delta], \widetilde{K}_{\bar{y}^{m}}(x)=\widetilde{K}\left(x, y_{1}, \ldots, y_{m}\right) \in \widetilde{L_{1}} M_{m}=\widetilde{L} M_{m}$ be delta-like kernels, defined and non-negative on the set $\Pi_{1, m}^{+}$and such that for all $\bar{y}>\overline{0}$ and $0<\delta<\pi$ it holds

$$
\int_{[-\pi, \pi)} \widetilde{K}_{\bar{y}^{m}}(x) d x=1, \quad \lim _{\bar{y} \rightarrow \overline{0}+\overline{0}} \int_{[-\pi, \pi) \backslash Q_{\delta}}\left|\widetilde{K}_{\bar{y}^{m}}(x)\right| d x=0 .
$$

Note that approximative properties of $2 \pi$-periodic analogs of these kernels were considered in the papers $[9,10]$.

Denote by

$$
\begin{aligned}
& \left\{\widetilde{X} * \widetilde{K}_{\bar{y}^{m}}(x)\right\}=\left\{\widetilde{u}(x, y)=\widetilde{u}(x, \bar{y})=\left(\widetilde{f} * \widetilde{K}_{\bar{y}^{m}}\right)(x)=\int_{[-\pi, \pi)} \widetilde{f}(x-t) \widetilde{K}_{\bar{y}^{m}}(t) d t:(\widetilde{f} \in \widetilde{X})\right\}, \\
& \left\{\overline{\widetilde{X} * \widetilde{K}_{\bar{y}^{m}}(x)}\right\}=\left\{\widetilde{v}(x, y)=\widetilde{v}(x, \bar{y})=\left\{\begin{array}{ll}
\left(\widetilde{f} * \widetilde{K}_{\bar{y}^{m}}\right)(x), & \bar{y}>\overline{0}, \\
\widetilde{f}(x), & \bar{y}=\overline{0},
\end{array}:(\widetilde{f} \in \widetilde{X})\right\}\right.
\end{aligned}
$$

the spaces of convolutions with delta-like kernels $\widetilde{K}_{\bar{y}^{m}}(x)$, where $\widetilde{X}$ is one of the spaces $\widetilde{C}, \widetilde{L}_{\infty}$, and $\widetilde{L}_{p}$. 
From the criteria of best approximation for the element $\widetilde{f} \in \widetilde{X}$ by elements of the subspace of all trigonometric polynomials $F_{2 n-1}$ of degree at most $n-1$ (see, e.g. [11, pp. 46-53]) follow the criteria of best approximation of isometric image $\widetilde{f} * \widetilde{K}_{\bar{y}^{m}}$ by the elements of isometric space $\left\{\overline{F_{2 n-1} * \widetilde{K}_{\bar{y}^{m}}}\right\}$.

An element $\psi T_{n-1}^{*}(x, y) \in\left\{\overline{F_{2 n-1} * \widetilde{K}_{\bar{y}^{m}}}\right\}$ is the element of best approximation of the element $\widetilde{v}(x, y)=\widetilde{f} * \widetilde{K}_{\bar{y}^{m}}(x)$ in the space $\widetilde{X} \bar{M}_{m}$ if and only if the trigonometric polynomial $T_{n-1}^{*}$ is the polynomial of best approximation of function $\widetilde{f} \in \widetilde{X}$ in the space $\widetilde{X}$. Hence, $E_{n}(\widetilde{v})_{\widetilde{C} \bar{M}_{m}}=\left\|\widetilde{v}(x, y)-\psi T_{n-1}^{*}(x, y)\right\|_{\widetilde{C} \bar{M}_{m}}$ if and only if there exist $2 n$ points $x_{k}$ such that $0 \leq x_{1}<x_{2}<\cdots<x_{2 n}<2 \pi$, and that the difference

$$
\Delta(x, 0)=\widetilde{v}(x, 0)-\psi T_{n-1}^{*}(x, 0)=\widetilde{f}(x)-T_{n-1}^{*}(x)
$$

attains the maximum value (by its absolute value) $\|\Delta(x, 0)\|_{\widetilde{C}}=\left\|\widetilde{v}(x, y)-\psi T_{n-1}^{*}(x, y)\right\|_{\widetilde{c} \bar{M}_{m}{ }^{\prime}}$ changing the sign one by one, i.e.,

$$
\Delta\left(x_{1}, 0\right)=-\Delta\left(x_{2}, 0\right)=\Delta\left(x_{3}, 0\right)=\cdots=-\Delta\left(x_{2 n}, 0\right)= \pm\|\Delta(x, 0)\|_{\widetilde{C}^{\prime}}
$$

and for $1<p<\infty$ the equality $E_{n}(\widetilde{v})_{\widetilde{p} \bar{M}_{m}}=\left\|\widetilde{v}(x, y)-\psi T_{n-1}^{*}(x, y)\right\|_{\widetilde{p} \bar{M}_{m}}$ holds if and only if for each element $\psi T_{n-1}^{*}(x, 0) \in\left\{\overline{F_{2 n-1} * \widetilde{K}_{\bar{y}^{m}}}\right\}$ the relation

$$
\int_{0}^{2 \pi} \psi T_{n-1}(x, 0)\left|\widetilde{v}(x, 0)-\psi T_{n-1}^{*}(x, 0)\right|^{p-1} \operatorname{sgn}\left(\widetilde{v}(x, 0)-\psi T_{n-1}^{*}(x, 0)\right) d x=0
$$

is true, where $\psi T_{n-1}(x, 0)=T_{n-1}(x)$ and $\psi T_{n-1}^{*}(x, 0)=T_{n-1}^{*}(x)$.

To approximate functions and classes of non-periodic functions, the entire functions of exponential type are used instead of trigonometric polynomials. To give examples of equality of approximation characteristics in the spaces of functions, isometric spaces of non-periodic functions, which are given by convolutions with entire functions of exponential type, it is necessary to establish integral representations for these convolutions.

\section{The spaces of convolutions with entire functions of exponential type and their integral representations}

Denote by $C^{n}, L_{\infty}^{n}, L_{\bar{p}}^{n}, \widehat{L}_{\bar{p}}^{n}$ the spaces of real functions, given on $E^{n}$, and, respectively, continuous and bounded, essentially bounded and measurable, with the norms

$$
\begin{gathered}
\|f\|_{C^{n}}=\sup _{x \in E^{n}}|f(x)|, \quad\|f\|_{\infty^{n}}=\sup _{x \in E^{n}} \operatorname{vrai}|f(x)|, \\
\|f\|_{\bar{p}^{n}}=\|\ldots\|\left\|f\left(x_{1}, \ldots x_{n}\right)\right\|_{p_{1}, x_{1}}\left\|_{p_{2}, x_{2}} \ldots\right\|_{p_{n}, x_{n}} \\
=\left[\int_{-\infty}^{\infty}\left[\ldots\left[\int_{-\infty}^{\infty}\left|f\left(x_{1}, x_{2}, \ldots, x_{n}\right)\right|^{p_{1}} d x_{1}\right]^{p_{2} / p_{1}} \ldots\right]^{p_{n} / p_{n-1}} d x_{n}\right]^{1 / p_{n}}, \\
\|f\|_{\hat{p}^{n}}=\|\ldots\|\left\|f\left(x_{1}, \ldots x_{n}\right)\right\|_{\widehat{p}_{1}, x_{1}}\left\|_{\widehat{p}_{2}, x_{2}} \ldots\right\|_{\hat{p}_{n}, x_{n}} \\
=\sup _{a_{n} \in E}\left[\int_{a_{n}}^{a_{n}+2 \pi}\left[\ldots \sup _{a_{1} \in E}\left[\int_{a_{1}}^{a_{1}+2 \pi}\left|f\left(x_{1}, \ldots, x_{n}\right)\right|^{p_{1}} d x_{1}\right]^{p_{2} / p_{1}} \ldots\right]^{p_{n} / p_{n-1}} d x_{n}\right]^{1 / p_{n}} .
\end{gathered}
$$


Let $X^{n}$ be one of the spaces $C^{n}, L_{\infty}^{n}, L_{\bar{p}}^{n}, \widehat{L}_{\bar{p}}^{n}(\overline{1} \leq \bar{p}<\bar{\infty})$ of (1)-(3),

$$
F_{\bar{\sigma}}^{X^{n}}=\left\{T_{\bar{\sigma}^{n}}(z)=T_{\bar{\sigma}^{n}}\left(x_{1}+i t_{1}, \ldots, x_{n}+i t_{n}\right): T_{\bar{\sigma}^{n}}(x) \in X^{n}\right\}
$$

are the spaces of entire functions of exponential type not greater than $\bar{\sigma}^{n}=\left(\sigma_{1}, \ldots, \sigma_{n}\right)$ (e.f.e.t.n.g. $\bar{\sigma}^{n}$ ) (see [2, pp. 118-119]) that belong to the space $X^{n}$ on the real $n$-dimensional Euclidean space $E^{n}, F_{\bar{\sigma}}^{L_{p}^{n}}=F_{\bar{\sigma}}^{p^{n}}, F_{\bar{\sigma}}^{L_{\bar{p}}^{n}}=F_{\bar{\sigma}}^{\bar{p}^{n}}, F_{\bar{\sigma}}^{\widehat{L}_{\bar{p}}^{n}}=F_{\bar{\sigma}}^{\widehat{\bar{p}}^{n}}, F_{\bar{\sigma}}^{L_{\infty}^{n}}=F_{\bar{\sigma}}^{\infty^{n}}, X^{n} \supset F_{\bar{\sigma}}^{X^{n}}\left(E^{n}\right)=\left\{T_{\bar{\sigma}^{n}}(x)\right.$ : $\left.T_{\bar{\sigma}^{n}}(z) \in F_{\bar{\sigma}}^{X^{n}}\right\}$ are their restrictions on the space $E^{n}, I_{\bar{y}^{m}}^{n}(x)$ are delta-like kernels (9), (10) from [4], defined on $\Pi_{n, m}^{+}$,

$$
\begin{gathered}
\left\{F_{\bar{\sigma}^{n}}^{X^{n}} * I_{\bar{y}^{m}}^{n}\right\}=\left\{\left(T_{\bar{\sigma}^{n}} * I_{\bar{y}^{m}}^{n}\right)(z)=\int_{E^{n}} I_{\bar{y}^{m}}^{n}(t) T_{\bar{\sigma}^{n}}(z-t) d t:\left(T_{\bar{\sigma}^{n}} \in F_{\bar{\sigma}^{n}}^{X^{n}}\right) \wedge(\bar{y}>\overline{0})\right\}, \\
\left\{\overline{F_{\bar{\sigma}}^{X^{n}} * I_{\bar{y}^{m}}^{n}}\right\}=\left\{I T_{\bar{\sigma}^{n}}(z, \bar{y})=\left\{\begin{array}{ll}
\left(T_{\bar{\sigma}^{n}} * I_{\bar{y}^{m}}^{n}\right)(z), & \bar{y}>\overline{0}, \\
T_{\bar{\sigma}^{n}}(z), & \bar{y}=\overline{0},
\end{array}:\left(T_{\bar{\sigma}^{n}} \in F_{\bar{\sigma}^{n}}^{X^{n}}\right)\right\}\right.
\end{gathered}
$$

are the spaces of convolutions of e.f.e.t.n.g. $\bar{\sigma}^{n}$ with delta-like kernels,

$$
\begin{gathered}
\left\{F_{\bar{\sigma}}^{X^{n}}\left(E^{n}\right) * I_{\bar{y}^{m}}^{n}\right\}=\left\{\left(T_{\bar{\sigma}^{n}} * I_{\bar{y}^{m}}^{n}\right)(x):\left(T_{\bar{\sigma}^{n}}(x) \in F_{\bar{\sigma}}^{X^{n}}\left(E^{n}\right)\right) \wedge(\bar{y}>\overline{0})\right\}, \\
\left\{\overline{F_{\bar{\sigma}}^{X^{n}}\left(E^{n}\right) * I_{\bar{y}^{m}}^{n}}\right\}=\left\{I T_{\bar{\sigma}^{n}}(x, y)=\left\{\begin{array}{ll}
\left(T_{\bar{\sigma}^{n}} * I_{\bar{y}^{m}}^{n}\right)(x), & \bar{y}>\overline{0}, \\
T_{\bar{\sigma}^{n}}(x), & \bar{y}=\overline{0},
\end{array}:\left(T_{\bar{\sigma}^{n}}(x) \in F_{\bar{\sigma}}^{X^{n}}\left(E^{n}\right)\right)\right\}\right.
\end{gathered}
$$

are their restrictions on $E^{n}$. If $n=1$, then we omit index $n$ and dashes over the vectors $\bar{\sigma}, \bar{p}, \overline{1}$, $\bar{\infty}$. In $[7,8]$ it was proved, that for each fixed $\bar{y}>\overline{0}$, the convolution of trigonometric polynomial with delta-like kernel is a trigonometric polynomial. An analogical statement holds true also for convolutions of entire functions with delta-like kernels.

Theorem 1. Arbitrary function from the space of convolutions (4) for each fixed $\bar{y}>\overline{0}$ belongs to the space $F_{\bar{\sigma}}{ }^{n}$.

Proof. Let $f \in L^{n}$, and $T_{\bar{\sigma}^{n}} \in F_{\bar{\sigma}}^{X^{n}}$. Let us show that the function

$$
\left(f * T_{\bar{\sigma}^{n}}\right)(z)=\int_{E^{n}} f(t) T_{\bar{\sigma}^{n}}(z-t) d t \in F_{\bar{\sigma}}^{X^{n}} .
$$

In the case $\overline{1}=(1, \ldots, 1) \leq \bar{p}=(p, \ldots, p) \leq \bar{q}=\left(q_{1}, \ldots, q_{n}\right) \leq \bar{\infty}=(\infty, \ldots, \infty)$, we have (see [13, pp. 153-155])

$$
F_{\bar{\sigma}}^{\bar{p}^{n}} \subseteq F_{\bar{\sigma}}^{\bar{q}^{n}} \subseteq F_{\bar{\sigma}}^{\infty^{n}}=F_{\bar{\sigma}}^{C^{n}}
$$

We want to prove that for $\overline{1} \leq \bar{p} \leq \bar{\infty}$ the equality

$$
F_{\overline{\bar{\sigma}}}^{\hat{\bar{p}}^{n}}=F_{\bar{\sigma}}^{C^{n}}
$$

is true.

For each function $T_{\bar{\sigma}^{n}} \in F_{\bar{\sigma}}^{C^{n}}$, in view of the norm definition of the spaces $\widehat{L}_{\bar{p}}^{n}$ and $C^{n}$, we have

$$
\left\|T_{\bar{\sigma}^{n}}\right\|_{\hat{\bar{p}}^{n}} \leq \prod_{i=1}^{n}(2 \pi)^{1 / p_{i}}\left\|T_{\bar{\sigma}^{n}}\right\|_{C^{n}}
$$


Now we show that

$$
\left\|T_{\bar{\sigma}^{n}}\right\|_{C^{n}} \leq \prod_{i=1}^{n}(2 \pi)^{1 / q_{i}}\left(\sigma_{i}^{1 / p_{i}}+1\right)\left\|T_{\bar{\sigma}^{n}}\right\|_{\overline{\bar{p}}^{n}},
$$

where $T_{\bar{\sigma}^{n}}$ is e.f.e.t.n.g. $\bar{\sigma}^{n}=\left(\sigma_{1}, \ldots, \sigma_{n}\right), \bar{p}=\left(p_{1}, \ldots, p_{n}\right)$ and $1 / p_{i}+1 / q_{i}=1(i=\overline{1, n})$.

To simplify the notations, we assume that $n=2$. It is known, see [12, pp. 191-192], that for each function $T_{\sigma} \in F_{\sigma}^{\widehat{p}}$ the following inequality holds

$$
\left\|T_{\sigma}\right\|_{C} \leq(2 \pi)^{1 / q}\left\|T_{\sigma}\right\|_{\widehat{p}}\left(\sigma^{1 / p}+1\right)
$$

where $1 / p+1 / q=1$. Taking into account that for each fixed $z_{1}$, the function $T_{\bar{\sigma}^{2}}\left(z_{1}, z_{2}\right)$ is (e.f.e.t.n.g. $\sigma_{2}$ ) in the variable $z_{2}$, then, using (12), we get

$$
\sup _{x_{2} \in E}\left|T_{\bar{\sigma}^{2}}\left(x_{1}, x_{2}\right)\right| \leq(2 \pi)^{1 / q_{2}}\left(\sigma_{2}^{1 / p_{2}}+1\right)\left\|T_{\bar{\sigma}^{2}}\left(x_{1}, x_{2}\right)\right\|_{\widehat{p}_{2}, x_{2}}
$$

where $1 / p_{2}+1 / q_{2}=1$. Then, by virtue of the inequality (13) and norm definition of the space $\widehat{L}_{\bar{p}}^{n}$, we get

$$
\begin{aligned}
\left\|T_{\bar{\sigma}^{2}}\left(x_{1}, x_{2}\right)\right\|_{C^{2}}=\sup _{x_{1} \in E} \sup _{x_{2} \in E}\left|T_{\bar{\sigma}^{2}}\left(x_{1}, x_{2}\right)\right| & \leq(2 \pi)^{1 / q_{2}}\left(\sigma_{2}^{1 / p_{2}}+1\right)\left\|\sup _{x_{1} \in E}\left|T_{\bar{\sigma}^{2}}\left(x_{1}, x_{2}\right)\right|\right\|_{\widehat{p}_{2}, x_{2}} \\
& \leq \prod_{i=1}^{2}(2 \pi)^{1 / q_{i}}\left(\sigma_{i}^{1 / p_{i}}+1\right)\|\| T_{\bar{\sigma}^{2}}\left(x_{1}, x_{2}\right)\left\|_{\widehat{p}_{1}, x_{1}}\right\|_{\widehat{p}_{2}, x_{2}} \\
& =\prod_{i=1}^{2}(2 \pi)^{1 / q_{i}}\left(\sigma_{i}^{1 / p_{i}}+1\right)\left\|T_{\bar{\sigma}^{2}}\left(x_{1}, x_{2}\right)\right\|_{\overline{\bar{p}}^{2}},
\end{aligned}
$$

where $1 / p_{i}+1 / q_{i}=1(i=\overline{1,2})$. Hence, the inequality (11) is true. From (10) and (11), we obtain (9). Theorem 3.6.2 in [2, p. 162] yields, that if $T_{\bar{\sigma}^{n}} \in F_{\bar{\sigma}}^{C^{n}}$, and $f \in L^{n}$, then

$$
\left(f * T_{\bar{\sigma}^{n}}\right) \in F_{\bar{\sigma}}^{C^{n}} .
$$

In the case $f \in L^{n}$, and $T_{\bar{\sigma}^{n}} \in F_{\bar{\sigma}^{n}}$, we have, in view of the relations (8) and (9), that the function $T_{\bar{\sigma}^{n}} \in F_{\bar{\sigma}}^{C^{n}}$, and from (14) get that $\left(f * T_{\bar{\sigma}^{n}}\right)$ is e.f.e.t.n.g. $\bar{\sigma}^{n}$. Using the generalized Minkovskyi inequality (see [4, p. 18]) and the norm invariance of the space $X^{n}$ with respect to the shift, we get

$$
\left\|f * T_{\bar{\sigma}^{n}}\right\|_{X^{n}}=\left\|\int_{E^{n}} f(t) T_{\bar{\sigma}^{n}}(x-t) d t\right\|_{X^{n}} \leq\left\|T_{\bar{\sigma}^{n}}(x-t)\right\|\left\|_{X^{n}}\right\| f\left\|_{L^{n}}=\right\| T_{\bar{\sigma}^{n}}\left\|_{X^{n}}\right\| f \|_{L^{n}},
$$

i.e. the function $\left(f * T_{\bar{\sigma}^{n}}\right)(x) \in X^{n}$.

Therefore, the relation ( 7 ) is true, and Theorem 1 is proved.

Let us prove that

$$
F_{\bar{\sigma}}^{\hat{\bar{p}}^{n}}\left(E^{n}\right)=F_{\bar{\sigma}}^{\infty^{n}}\left(E^{n}\right)=F_{\bar{\sigma}}^{C^{n}}\left(E^{n}\right) \subset C_{r}^{n},
$$

where $C^{n} \supset C_{r}^{n}$ is a subspace of uniformly continuous functions from the space $C^{n}$. In view of the fact that the equality $F_{\bar{\sigma}}^{\hat{\bar{p}}^{n}}=F_{\bar{\sigma}}^{\infty}=F_{\bar{\sigma}}^{C^{n}}$ was established in proving Theorem 1 , it is sufficient to show that $F_{\bar{\sigma}}^{C^{n}}\left(E^{n}\right) \subset C_{r}^{n}$. To simplify the notations, we assume that $n=2$. Let $T_{\bar{\sigma}^{2}}(x, y)$ be an arbitrary function from the space $F_{\bar{\sigma}} C^{2}\left(E^{2}\right)$. The function $T_{\bar{\sigma}^{2}}(x, y)$ is differentiable in each 
variable. Therefore, for each point $M_{1}\left(x_{1}, y_{1}\right)$ i $M_{2}\left(x_{2}, y_{2}\right)$ of the space $E^{2}$, using the Lagrange theorem, we have

$$
\begin{aligned}
\left|T_{\bar{\sigma}^{2}}\left(x_{1}, y_{1}\right)-T_{\bar{\sigma}^{2}}\left(x_{2}, y_{2}\right)\right| & \leq\left|T_{\bar{\sigma}^{2}}\left(x_{1}, y_{1}\right)-T_{\bar{\sigma}^{2}}\left(x_{2}, y_{1}\right)\right|+\left|T_{\bar{\sigma}^{2}}\left(x_{2}, y_{1}\right)-T_{\bar{\sigma}^{2}}\left(x_{2}, y_{2}\right)\right| \\
& =\left|\left(T_{\bar{\sigma}^{2}}\left(\zeta, y_{1}\right)\right)_{x}^{\prime}\right|\left|x_{2}-x_{1}\right|+\left|\left(T_{\bar{\sigma}^{2}}\left(x_{2}, \eta\right)\right)_{y}^{\prime}\right|\left|y_{2}-y_{1}\right|,
\end{aligned}
$$

where $\zeta \in\left(x_{1}, x_{2}\right)$ and $\eta \in\left(y_{1}, y_{2}\right)$.

For each fixed $z_{2}$, the function $T_{\bar{\sigma}^{2}}\left(z_{1}, z_{2}\right)$ is e.f.e.t.n.g. $\sigma_{1}$ in variable $z_{1}$, and for each fixed $z_{1}$ it is e.f.e.t.n.g. $\sigma_{2}$. Then, in view of the Bernstein inequality (see, e.g., [2, p. 138]), we get

$$
\left\|\left(T_{\bar{\sigma}^{2}}(x, y)\right)_{x}^{\prime}\right\|_{C^{2}} \leq \sigma_{1}\left\|T_{\bar{\sigma}^{2}}(x, y)\right\|_{C^{2},} \quad\left\|\left(T_{\bar{\sigma}^{2}}(x, y)\right)_{y}^{\prime}\right\|_{C^{2}} \leq \sigma_{2}\left\|T_{\bar{\sigma}^{2}}(x, y)\right\|_{C^{2}} .
$$

The inequalities (16) and (17), in combination with the inequalities between means, yield

$$
\begin{aligned}
\left|T_{\bar{\sigma}^{2}}\left(x_{1}, y_{1}\right)-T_{\bar{\sigma}^{2}}\left(x_{2}, y_{2}\right)\right| & \leq\left\|T_{\bar{\sigma}^{2}}\right\|_{C^{2}}\left(\sigma_{1}\left|x_{2}-x_{1}\right|+\sigma_{2}\left|y_{2}-y_{1}\right|\right) \\
& \leq \sqrt{2} \max \left\{\sigma_{1}, \sigma_{2}\right\}\left\|T_{\bar{\sigma}^{2}}\right\|_{C^{2}} \sqrt{\left(x_{1}-x_{2}\right)^{2}+\left(y_{1}-y_{2}\right)^{2}} .
\end{aligned}
$$

From (18) we get, that the function $T_{\bar{\sigma}^{2}}(x, y)$ is uniformly continuous on $E^{2}$. It means that the relations (15) are true.

Therefore, if $X^{n}$ is one of the spaces $C^{n}, L_{\infty}^{n}, L_{\bar{p}}^{n}, \widehat{L}_{\bar{p}}^{n}(\overline{1} \leq \bar{p}<\bar{\infty})$ then Corollary 1 from [4] in combination with (8) and (15) yields that the spaces $\left\{F_{\bar{\sigma}}^{X^{n}}\left(E^{n}\right) * I_{\bar{y}^{m}}^{n}\right\}$ and $\left\{\overline{F_{\bar{\sigma}}^{X^{n}}\left(E^{n}\right) * I_{\bar{y}^{m}}^{n}}\right\}$ are isomorphic to the space $\left\{F_{\bar{\sigma}}^{X^{n}}\left(E^{n}\right)\right\}$ and isomertic to this space in the case when the kernel $I_{\bar{y}^{m}}^{n}$ is non-negative.

Let us find an integral representation of functions from the spaces (5) and (6). Denote with $\Delta_{\bar{\sigma}}^{n}=\left[-\sigma_{1}, \sigma_{1}\right] \times \ldots \times\left[-\sigma_{n}, \sigma_{n}\right] \subset E^{n} n$-dimensional parallelepiped of the space $E^{n}, L_{p\left(\Delta_{\bar{\sigma}}\right)}$ the space of measuable real or complex functions, the $p$ th modulus of which is summable, with the norm

$$
\|f\|_{L_{p\left(\Delta \frac{n}{\sigma}\right)}}=\|f\|_{p\left(\Delta \frac{n}{\sigma}\right)}=\left(\int_{\Delta_{\bar{\sigma}}^{n}}|f(x)|^{p} d x\right)^{1 / p} .
$$

Theorem 2. The following relations hold:

$$
\begin{gathered}
\left\{F_{\bar{\sigma}}^{2^{n}}\left(E^{n}\right) * I_{\bar{y}^{m}}^{n}\right\}=\left\{\left(T_{\bar{\sigma}^{n}} * I_{\bar{y}^{m}}^{n}\right)(x)=\int_{\Delta_{\bar{\sigma}}^{n}} F\left(I_{\bar{y}^{m}}^{n}\right)(-t) \varphi(t) e^{i t x} d t:\left(\varphi \in L_{2\left(\Delta_{\bar{\sigma}}^{n}\right)}\right) \wedge(\bar{y}>\overline{0})\right\}, \\
\left\{\overline{\bar{F}_{\bar{\sigma}}^{n}\left(E^{n}\right) * I_{\bar{y}^{m}}^{n}}\right\}=\left\{I T_{\bar{\sigma}^{n}}(x, y)=\left\{\begin{array}{ll}
\left(T_{\bar{\sigma}^{n}} * I_{\bar{y}^{m}}^{n}\right)(x), & \bar{y}>\overline{0}, \\
\int_{\Delta_{\bar{\sigma}}^{n}} \varphi(t) e^{i t x} d t, & \bar{y}=\overline{0},
\end{array}:\left(\varphi \in L_{2\left(\Delta \frac{n}{\bar{\sigma}}\right)}\right)\right\},\right.
\end{gathered}
$$

where

$$
F\left(I_{\bar{y}^{m}}^{n}\right)(x)=\int_{E^{n}} I_{\bar{y}^{m}}^{n}(t) e^{i t x} d t
$$

is the fourier transform of the function $I_{\bar{y}^{m}}^{n}$.

Proof. By the Wiener-Paley theorem (see [12, pp. 130-131]),

$$
F_{\bar{\sigma}}^{2^{n}}=\left\{T_{\bar{\sigma}^{n}}(z)=\int_{\Delta_{\bar{\sigma}}^{n}} \varphi(t) e^{i t x} d t:\left(\varphi \in L_{2\left(\Delta \frac{n}{\sigma}\right)}\right)\right\}
$$


and the definitions (5), (6). Since the function $T_{\bar{\sigma}^{n}} \in L_{2\left(\Delta \frac{n}{\sigma}\right)}$, then, by (55) from [4] and the change of variables, we have for almost all $t \in E^{n}$

$$
F\left(T_{\bar{\sigma}^{n}}\right)(t)=(2 \pi)^{n} F^{-1}\left(T_{\bar{\sigma}^{n}}\right)(-t)=(2 \pi)^{n} \varphi(-t) .
$$

In view of the fact that for all $\bar{y}>\overline{0}$ the kernel $I_{\bar{y}^{m}}^{n} \in L^{n}$, we get that the function $F\left(I_{\bar{y}^{m}}^{n}\right)$ is uniformly continuous and bounded on $E^{n}$. Since the function $\varphi(t) \in L_{2\left(\Delta_{\bar{\sigma}}\right)}$, then

$$
\left|F\left(I_{\bar{y}^{m}}^{n}\right)(-t) \varphi(t)\right| \in L^{n}
$$

Hence, by Lemma 7 from [4], using (21), (22) and changing the variables, for all $x \in E^{n}$ the following equality holds

$$
\left(T_{\bar{\sigma}^{n}} * I_{\bar{y}^{m}}^{n}\right)(x)=\int_{\Delta_{\bar{\sigma}}^{n}} F\left(I_{\bar{y}^{m}}^{n}\right)(-t) \varphi(t) e^{i t x} d t
$$

The equality (23) yields (19).

Corollary 1. The following relations hold:

$$
\begin{gathered}
\left\{F_{\bar{\sigma}}^{2^{n}}\left(E^{n}\right) * I_{\bar{y}^{m}}^{n}\right\}=\left\{\left(T_{\bar{\sigma}^{n}} * I_{\bar{y}^{m}}^{n}\right)(x)=\int_{\Delta_{\bar{\sigma}}^{n}} F\left(I_{\bar{y}^{m}}^{n}\right)(-t)(a(t)+i b(t)) e^{i t x} d t:\right. \\
\left.\left(a(t) \in L_{2\left(\Delta \frac{n}{\sigma}\right)}\right) \wedge\left(b(t) \in L_{2\left(\Delta \frac{n}{\sigma}\right)}\right) \wedge(\bar{y}>\overline{0})\right\}, \\
\left\{\overline{F_{\bar{\sigma}^{n}}\left(E^{n}\right) * I_{\bar{y}^{m}}^{n}}\right\} \\
= \begin{cases}\left(T_{\bar{\sigma}^{n}}(x, y)=\left\{\begin{array}{ll}
\left(T_{\bar{\sigma}^{n}} * I_{\bar{y}^{m}}^{n}\right)(x), & \bar{y}>\overline{0}, \\
\int_{\Delta_{\bar{\sigma}}^{n}}(a(t)+i b(t)) e^{i t x} d t, & \bar{y}=\overline{0},
\end{array}:\left(a(t) \in L_{2\left(\Delta \frac{n}{\sigma}\right)}\right) \wedge\left(b(t) \in L_{2\left(\Delta \frac{n}{\sigma}\right)}\right)\right\},\right.\end{cases}
\end{gathered}
$$

where $a(t)$ is even $b(t)$ is od in each variable $t_{1}, \ldots, t_{n}$ are real functions.

Proof. Since the functions from the space (20) are real on $\Pi_{n, m}^{+}$, then the function

$$
\operatorname{IT}_{\bar{\sigma}^{n}}(x, 0)=T_{\bar{\sigma}^{n}}(x)=\int_{\Delta \frac{n}{\sigma}} \varphi(t) e^{i t x} d t
$$

is real on $E^{n}$. Therefore, it coincides with the conjugate function, i.e.,

$$
\overline{T_{\bar{\sigma}^{n}}}=\int_{\Delta_{\bar{\sigma}}^{n}} \bar{\varphi}(t) e^{-i t x} d t=\int_{\Delta_{\bar{\sigma}}^{n}}(a(t)-i b(t)) e^{-i t x} d t=\int_{\Delta_{\bar{\sigma}}^{n}}(a(-t)-i b(-t)) e^{i t x} d t,
$$

where $a(t)$ and $b(t)$ are real functions from the space $L_{2\left(\Delta \frac{n}{\sigma}\right)}$. In the last equality we used the change of variables. The relations (26), taking into account the formulas of rotation (see $[4$, p. 55]) and changing the variables, we get

$$
a(t)=a(-t), \quad b(t)=-b(-t) .
$$

The equalities (27) yield, that the function $a(t)$ is even, and $b(t)$ is odd in each variable $t_{1}, \ldots, t_{n}$. Then, from the relations (19), (20) we obtain (24), (25), and Corollary 1 is proved. 
From Theorem 1, Corollary 1 and the relation (8) we get, that for

$$
\overline{1}(1, \ldots, 1) \leq \bar{p} \leq \overline{2}(2, \ldots, 2)
$$

and the spaces of convolutions $\left\{F_{\bar{\sigma}}^{\bar{p}^{n}}\left(E^{n}\right) * I_{\bar{y}}^{n}\right\}$ and $\left\{\overline{F_{\bar{\sigma}}^{\bar{p}^{n}}\left(E^{n}\right) * I_{\bar{y}^{m}}^{n}}\right\}$ the following integral representations hold true: (19), (20), (24), (25).

The functions from the space $F_{\sigma}^{C}$ satisfy the equalities (see, e.g., [2, p. 182])

$$
F_{\sigma}^{C}=\left\{g_{\sigma}(z)=g_{\sigma}(0)+z T_{\sigma}(z): T_{\sigma} \in F_{\sigma}^{2}\right\} .
$$

Let us find the integral representations for the functions from the space $\left\{F_{\sigma}^{C} * I_{\bar{y}^{m}}\right\}$ under the additional conditions on the kernel. The following statement holds.

Theorem 3. Let

$$
I_{\bar{y}^{m}}(x)=F^{-1}\left(\psi_{\bar{y}^{m}}(|u|)\right)(x)=\frac{1}{2 \pi} \int_{-\infty}^{\infty} \psi_{\bar{y}^{m}}(|u|) e^{-i u x} d u=\frac{1}{\pi} \int_{0}^{\infty} \psi_{\bar{y}^{m}}(u) \cos u x d u
$$

is a delta-like kernel, defined on $\Pi_{1, m^{\prime}}^{+}$and function $\psi_{\bar{y}^{m}}(|t|)=\psi(|t|, \bar{y})$ is locally absolutely continuous in the variable $t$ for each fixed $\bar{y}>\overline{0}$. Then for all $\bar{y}>\overline{0}$ the relation

$$
f_{\bar{y}^{m}}(x)=x I_{\bar{y}^{m}}(x)=\frac{i}{2 \pi} \int_{-\infty}^{\infty} \operatorname{sgn}(t) \psi_{t}^{\prime}(|t|, \bar{y}) e^{-i t x} d t=\frac{1}{2 \pi} \int_{-\infty}^{\infty} \operatorname{sgn}(t) \psi_{t}^{\prime}(|t|, \bar{y}) \sin t x d t
$$

holds. If for each $\bar{y}>\overline{0}$ the function $f_{\bar{y}^{m}}(x)$ belongs to the space $L$, or the function $\operatorname{sgn}(t) \psi_{t}^{\prime}(|t|, \bar{y})$ belongs to the space $L_{2}$, and the function $g_{\sigma}$ is in $F_{\sigma}^{C}$, then we have the relations:

$$
\begin{aligned}
&\left\{F_{\sigma}^{C}(E) * I_{\bar{y}^{m}}\right\}=\left\{\left(g_{\sigma} * I_{\bar{y}^{m}}\right)(x)=g_{\sigma}(0)+x \int_{-\sigma}^{\sigma} \psi_{\bar{y}^{m}}(|u|) \varphi(u) e^{i u x} d u\right. \\
&\left.-i \int_{-\sigma}^{\sigma} \operatorname{sgn}(u) \psi_{u}^{\prime}(|u|, \bar{y}) \varphi(u) e^{i u x} d u:\left(\varphi \in L_{2(-\sigma, \sigma)}\right) \wedge(\bar{y}>\overline{0})\right\}, \\
&\left\{\overline{F_{\sigma}^{C}(E) * I_{\bar{y}^{m}}}\right\}=\left\{g_{\sigma}(x, y)=\left\{\begin{array}{ll}
\left(g_{\sigma} * I_{\bar{y}^{m}}\right)(x), & \bar{y}>\overline{0}, \\
g_{\sigma}(x)=g_{\sigma}(0)+x T_{\sigma}(x), & \bar{y}=\overline{0}
\end{array}:\left(T_{\sigma} \in F_{\sigma}^{2}\right)\right\} .\right.
\end{aligned}
$$

Proof. The function $\psi_{\bar{y}^{m}}(|u|)$ is locally absolutely continuous. Then (see, e.g., [14, p. 229]) for all $\bar{y}>\overline{0}$ and almost all real $u$ it holds

$$
\psi_{u}^{\prime}(|u|, \bar{y})=\operatorname{sgn}(u) \psi_{u}^{\prime}(|u|, \bar{y}) .
$$

In view of (32) (see [4, p. 71]) and integrating by parts, that is possible due to the absolute integrability of the function $\psi_{\bar{y}^{m}}(|t|)$ in variable $t$, we get

$$
I_{\bar{y}^{m}}(x)=\frac{i}{2 \pi x} \int_{-\infty}^{\infty} \operatorname{sgn}(t) \psi_{t}^{\prime}(|t|, \bar{y}) e^{-i t x} d t .
$$

The relation (33) yields (29). If $h(x)=x T_{\sigma}(x)$, then from (28) we have that $g_{\sigma}(x)=g_{\sigma}(0)+$ $h(x)$ and, taking into account (29) and (9) from [4], making the change of variables, we get

$$
\begin{aligned}
\left(g_{\sigma} * I_{\bar{y}^{m}}\right)(x)= & \int_{-\infty}^{\infty} g_{\sigma}(t) I_{\bar{y}^{m}}(x-t) d t=g_{\sigma}(0)+x \int_{-\infty}^{\infty} T_{\sigma}(t) I_{\bar{y}^{m}}(x-t) d t \\
& -\int_{-\infty}^{\infty}(x-t) I_{\bar{y}^{m}}(x-t) T_{\sigma}(t) d t=g_{\sigma}(0)+x\left(T_{\sigma} * I_{\bar{y}^{m}}\right)(x)-\left(f_{\bar{y}^{m}} * T_{\sigma}\right)(x),
\end{aligned}
$$


where, in view of the relations $(21),(28), T_{\sigma}(x) \in F_{\sigma}^{2}$. Since the function

$$
I_{\bar{y}^{m}}(x)=F^{-1}\left(\psi_{\bar{y}^{m}}(|u|)\right)(x) \in L,
$$

then by (70) from [4], for all $\bar{y}>\overline{0}$ and real $t$ the relation $F\left(F^{-1}\left(\psi_{\bar{y}^{m}}\right)(t)=\psi_{\bar{y}^{m}}(t)\right.$ holds. By Theorem 2 , in the case $n=1$, we obtain

$$
\left(T_{\sigma} * I_{\bar{y}^{m}}\right)(x)=\int_{-\sigma}^{\sigma} \psi_{\bar{y}^{m}}(|t|) \varphi(t) e^{i t x} d t .
$$

If for each $\bar{y}>\overline{0}$ the function $f_{\bar{y}^{m}}(x)$ belongs to the space $L$, then by Lemma 7 from [4], using the equalities (21), (29), we get

$$
\left(f_{\bar{y}^{m}} * T_{\sigma}\right)(x)=i \int_{-\sigma}^{\sigma} \operatorname{sgn}(u) \psi_{u}^{\prime}(|u|, \bar{y}) \varphi(u) e^{i u x} d u .
$$

If for each $\bar{y}>\overline{0}$ the function $\operatorname{sgn}(u) \psi_{u}^{\prime}(|u|, \bar{y}) \in L_{2}$, then the relations (57) from [4], taking into account that $\varphi \in L_{2}$, yield the relation (36). From (19), (20), (34)-(36) we get (30), (31).

Since the functions from the spaces of convolutions (30), (31) should be real on the set $\Pi_{1, m}^{+}$, then, speculating in a similar way as in proving Corollary 1 , we derive to the following statement.

Corollary 2. The following relations hold

$$
\begin{aligned}
\left\{F_{\sigma}^{C}(E) * I_{\bar{y}^{m}}\right\}= & \left\{\left(g_{\sigma} * I_{\bar{y}^{m}}\right)(x)=g_{\sigma}(0)+2 x \int_{0}^{\sigma} \psi_{\bar{y}^{m}}(t)(a(t) \cos t x-b(t) \sin t x) d t\right. \\
+ & 2 \int_{0}^{\sigma} \psi_{t}^{\prime}(t, \bar{y})(a(t) \sin t x+b(t) \cos t x) d t: \\
& \left.\left(a(t) \in L_{2(-\sigma, \sigma)}\right) \wedge\left(b(t) \in L_{2(-\sigma, \sigma)}\right) \wedge(\bar{y}>\overline{0})\right\}, \\
\left\{\overline{F_{\sigma}^{C}(E) * I_{\bar{y}^{m}}}\right\}= & \left\{g_{\sigma}(x, y)\right. \\
= & \begin{cases}\left(g_{\sigma} * I_{\bar{y}^{m}}\right)(x), & \bar{y}>\overline{0}, \\
g_{\sigma}(x)=g_{\sigma}(0)+2 x \int_{0}^{\sigma}(a(t) \cos t x-b(t) \sin t x) d t, & \bar{y}=\overline{0},\end{cases} \\
& \left.\left(a(t) \in L_{2(-\sigma, \sigma)}\right) \wedge\left(b(t) \in L_{2(-\sigma, \sigma)}\right)\right\},
\end{aligned}
$$

where the kernel $I_{\bar{y}^{m}}(x)$ satisfies the conditions of Theorem 3, and $a(t)$ is an even, $b(t)$ is an odd function.

Theorems 2, 3, Corollaries 1, 2 and the relations (8), (9) yield, that for $1 \leq p \leq 2$ for the spaces of convolutions $\left\{F_{\sigma}^{p}(E) * I_{\bar{y}^{m}}\right\}$ and $\left\{\overline{F_{\sigma}^{p}(E) * I_{\bar{y}^{m}}}\right\}$ the respective integral representations hold (19), (20), (24), and (25). If the kernel $\left.I_{\bar{y}^{m}}\right)(x)$ satisfies the conditions of Theorem 3 , then for $2<p \leq \infty$ for these spaces of convolutions and for $1 \leq p \leq \infty$ for the spaces of convolutions $\left\{F_{\sigma}^{\widehat{p}}(E) * I_{\bar{y}^{m}}\right\}$ and $\left\{\overline{F_{\sigma}^{\widehat{p}}(E) * I_{\bar{y}^{m}}}\right\}$ we have, correspondingly, the integral representations (30), (31), (37), and (38).

To give examples of delta-like kernels, that satisfy or not the conditions of Theorem 3 , let us obtain the sufficient conditions for function $\Psi_{\bar{y}^{m}}(x)=F^{-1}\left(\psi_{\bar{y}^{m}}(|u|)\right)(x)$ to be a non-negative delta-like kernel, i.e., to satisfy the conditions of Corollary 6 from [4]. The following statement holds. 
Lemma 1. If for each $\bar{y}>\overline{0}$ the function $\psi_{\bar{y}^{m}}(|u|)$ is convex downward on the interval $(0, \infty)$,

$$
\psi_{\bar{y}^{m}}(0)=1, \quad \lim _{|u| \rightarrow \infty} \psi_{\bar{y}^{m}}(|u|)=0
$$

and for all real $u$ the equality

$$
\lim _{\bar{y} \rightarrow \overline{0}+\overline{0}} \psi_{\bar{y}^{m}}(|u|)=\lim _{\bar{y} \rightarrow \overline{0}+\overline{0}} \psi(|u|, \bar{y})=1
$$

is true, then the function $\Psi_{\bar{y}^{m}}(x)=F^{-1}\left(\psi_{\bar{y}^{m}}(|u|)\right)(x)$ is a non-negative delta-like kernel.

Proof. In view of the fact, that for all $\bar{y}>\overline{0}$ the function $\psi_{\bar{y}^{m}}(|u|)$ is convex downward on the interval $(0, \infty)$, from (39) we get that this function is bounded on $\Pi_{1, m}^{+}$. Then (see, e.g., [2, pp. 167-168]) for all $\bar{y}>\overline{0}$ the function $\Psi_{\bar{y}^{m}}(x)$ is non-negative, absolute integrable on the whole real axis and continuous possibly except of the point $x=0$. Then, in view of the equalities (39), (40) the functions $\Psi_{\bar{y}^{m}}(x)$ and $\psi_{\bar{y}^{m}}(|u|)$ satisfy the conditions of Corollary 6 from [4]. Hence, by Corollary 6, the function $\Psi_{\bar{y}^{m}}(x)$ is a non-negative delta-like kernel.

Corollary 3. If the function $\psi(|u|)$ is non-decreasing, convex upwards on the interval $(0, \infty)$,

$$
\psi(0)=0, \quad \lim _{|u| \rightarrow \infty} \psi(|u|)=\infty,
$$

and the function $\varphi(\bar{y})$ is positive on the set $\Pi_{0, m}^{+}=\left\{\bar{y} \in E^{m}: \bar{y}>\overline{0}\right\}$,

$$
\lim _{\bar{y} \rightarrow \overline{0}+\overline{0}} \varphi(\bar{y})=0 \text {, }
$$

then the function

$$
\Psi_{\varphi(\bar{y})}(x)=F^{-1}\left(e^{-\varphi(\bar{y}) \psi(|u|)}\right)(x)=\frac{1}{2 \pi} \int_{-\infty}^{\infty} e^{-\varphi(\bar{y}) \psi(|u|)} e^{-i u x} d u
$$

is a non-negative delta-like kernel.

Proof. From the definition of upward convexity, for all $u_{1}>0$ and $u_{2}>0$ it holds

$$
\psi\left(\frac{u_{1}+u_{2}}{2}\right) \geq \frac{\psi\left(u_{1}\right)+\psi\left(u_{2}\right)}{2} .
$$

Using the inequality (43), the arithmetic and geometric means theorem, and taking into account that the function $\varphi(\bar{y})$ is positive, we get

$$
\begin{aligned}
e^{-\varphi(\bar{y}) \psi\left(\left(u_{1}+u_{2}\right) / 2\right)} & \leq e^{-\varphi(\bar{y})\left(\psi\left(u_{1}\right)+\psi\left(u_{2}\right)\right) / 2} \\
& =\left(e^{-\varphi(\bar{y}) \psi\left(u_{1}\right)} e^{-\varphi(\bar{y}) \psi\left(u_{2}\right)}\right)^{1 / 2} \leq \frac{1}{2}\left(e^{-\varphi(\bar{y}) \psi\left(u_{1}\right)}+e^{-\varphi(\bar{y}) \psi\left(u_{2}\right)}\right) .
\end{aligned}
$$

From (44), by the definition of downward convexity, we have that for all $\bar{y}>\overline{0}$ the function $e^{-\varphi(\bar{y}) \psi(|u|)}$ is downward convex on the interval $(0, \infty)$. From (41) we get that for all $\bar{y}>\overline{0}$

$$
e^{-\varphi(\bar{y}) \psi(0)}=1, \quad \lim _{|u| \rightarrow \infty} e^{-\varphi(\bar{y}) \psi(|u|)}=0,
$$

and from (42) that for all real $u$ it holds

$$
\lim _{\bar{y} \rightarrow \overline{0}+\overline{0}} e^{-\varphi(\bar{y}) \psi(|u|)}=1 .
$$

Therefore, in view of the equalities (45) and (46), the function $e^{-\varphi(\bar{y})} \psi(|u|)$ satisfies the conditions of Lemma 1. Hence, by Lemma 1, the function $\Psi_{\varphi(\bar{y})}(x)$ is a non-negative delta-like kernel. 
Let us give examples of functions that satisfy the conditions of Corollary 3 and Theorem 3.

Example. Let the function $\varphi(\bar{y})$ satisfies the conditions of Corollary $3, \psi_{\alpha}(u)=|u|^{\alpha}, \psi_{\beta}(u)=$ $\ln ^{\beta}(1+|u|), \psi_{\gamma}(u)=\ln ^{\gamma} \ln (e+|u|)$ and $0<\alpha, \beta, \gamma \leq 1$, then we can check that the functions $\psi_{\alpha}(u), \psi_{\beta}(u), \psi_{\gamma}(u)$ satisfy the conditions of Corollary 3. Then, the functions

$$
\begin{gathered}
\Psi_{\varphi(\bar{y})}^{\alpha}(x)=\frac{1}{2 \pi} \int_{-\infty}^{\infty} e^{-\varphi(\bar{y})|u|^{\alpha}} e^{-i u x} d u=\frac{1}{\pi} \int_{0}^{\infty} e^{-\varphi(\bar{y})|u|^{\alpha}} \cos u x d u, \\
\Psi_{\varphi(\bar{y}), \beta}(x)=\frac{1}{2 \pi} \int_{-\infty}^{\infty} e^{-\varphi(\bar{y}) \ln ^{\beta}(1+|u|)} e^{-i u x} d u=\frac{1}{\pi} \int_{0}^{\infty} e^{-\varphi(\bar{y}) \ln ^{\beta}(1+u)} \cos u x d u, \\
\Psi_{\varphi(\bar{y}), \gamma}(x)=\frac{1}{2 \pi} \int_{-\infty}^{\infty} e^{-\varphi(\bar{y}) \ln ^{\gamma} \ln (e+|u|)} e^{-i u x} d u=\frac{1}{\pi} \int_{0}^{\infty} e^{-\varphi(\bar{y}) \ln ^{\gamma} \ln (e+u)} \cos u x d u
\end{gathered}
$$

are non-negative delta-like kernels.

If $\varphi(\bar{y})=y^{3} \beta=1$, then the kernel

$$
\Phi_{y}(x)=\frac{1}{2 \pi} \int_{-\infty}^{\infty} e^{-y \ln (1+|u|)} e^{-i u x} d u=\frac{1}{\pi} \int_{0}^{\infty} \frac{\cos u x}{(1+u)^{y}} d u
$$

(see, e.g., [14, p. 397]) coincides with the Flett kernel and is a non-negative delta-like kernel.

It is known (see, e.g., [3, pp. 115-116]) that if $1<\alpha \leq 2$ then the functions

$$
\Psi_{\alpha}(x)=\frac{1}{2 \pi} \int_{-\infty}^{\infty} e^{-|u|^{\alpha}} e^{-i u x} d u
$$

are non-negative and absolute integrable on the whole real axis. Making the change of variables, for all $y>0$ we have

$$
y^{-1 / \alpha} \Psi_{\alpha}\left(x y^{1 / \alpha}\right)=\frac{1}{2 \pi} \int_{-\infty}^{\infty} e^{-y|t|^{\alpha}} e^{-i t x} d t=\Psi_{y}^{\alpha}(x)
$$

Hence, for $0<\alpha \leq 2$ and $y>0$ the function $\Psi_{y}^{\alpha}(x)$ is non-negative and absolute integrable on the whole real axis, but for $1<\alpha \leq 2$ the function $\varphi_{\alpha}(u)=|u|^{\alpha}$ is convex downward but not upward.

Let us show, that if $0<\alpha, \beta, \gamma<1 / 2$ and $1=\beta=\gamma \leq \alpha \leq 2$ the non-negative delta-like kernels $\Psi_{y}^{\alpha}(x)$,

$$
\begin{gathered}
\Psi_{y, \beta}(x)=\frac{1}{2 \pi} \int_{-\infty}^{\infty} e^{-y \ln ^{\beta}(1+|u|)} e^{-i u x} d u=\frac{1}{\pi} \int_{0}^{\infty} e^{-y \ln ^{\beta}(1+u)} \cos u x d u, \\
\Psi_{y, \gamma}(x)=\frac{1}{2 \pi} \int_{-\infty}^{\infty} e^{-y \ln ^{\gamma} \ln (e+|u|)} e^{-i u x} d u=\frac{1}{\pi} \int_{0}^{\infty} e^{-y \ln \gamma \ln (e+u)} \cos u x d u
\end{gathered}
$$

satisfy the conditions of Theorem 3 , and for $1 / 2 \leq \alpha, \beta, \gamma<1$ they do not satisfy these conditions.

If $1=\beta=\gamma \leq \alpha \leq 2$, then for all $y>0$ we can check that the functions

$$
\begin{aligned}
\left(e^{-y|u|^{\alpha}}\right)_{u}^{\prime} & =-y \alpha \operatorname{sgn}(u)|u|^{\alpha-1} e^{-y|u|^{\alpha}}, \\
\left(e^{-y \psi_{\beta}(|u|)}\right)_{u}^{\prime} & =\left(e^{-y \ln ^{\beta}(1+|u|)}\right)_{u}^{\prime}=e^{-y \ln ^{\beta}(1+|u|)}\left[-y \beta \operatorname{sgn}(u) \frac{\ln ^{\beta-1}(1+|u|)}{1+|u|}\right], \\
\left(e^{-y \psi_{\gamma}(|u|)}\right)_{u}^{\prime} & =\left(e^{-y \ln ^{\gamma} \ln (e+|u|)}\right)_{u}^{\prime}=e^{-y \ln ^{\gamma} \ln (e+|u|)}\left[-y \gamma \operatorname{sgn}(u) \frac{\ln ^{\gamma-1} \ln (e+|u|)}{(e+|u|) \ln (e+|u|}\right]
\end{aligned}
$$


belong to the space $L_{2}$, and delta-like kernels $\Psi_{y}^{\alpha}(x), \Psi_{y, \beta}(x)$ and $\Psi_{y, \gamma}(x)$ satisfy the conditions of Theorem 3.

So, the function $f(u)=\ln (1+u)$ is convex upward on the interval $(0, \infty), f(0)=0$ and $f^{\prime}(0)=1$, then for $u \geq 0$ the inequality $\ln (1+u) \leq u$ holds. In view of $f(0)=0$ and $f(e-1)=1$, then, taking into account an upward convexity of $f(u)$, for $0 \leq u \leq e-1$ it holds $\ln (1+u) \geq u /(e-1)$. The, in the case $0 \leq u \leq e-1$ we get

$$
\frac{u}{e-1} \leq \ln (1+u) \leq u \text {. }
$$

Similarly, one can prove that for $0 \leq u \leq e^{e}-e$ the following inequality holds

$$
\frac{u}{e^{e}-e} \leq \ln (\ln (e+u)) \leq \frac{u}{e}
$$

Form (48), (49) we get that for all $\delta>0$ and $0<u \leq e-1$ the inequalities

$$
e^{\delta} u^{-\delta}<\ln ^{-\delta} \ln (e+u)<\left(e^{e}-e\right)^{\delta} u^{-\delta}, u^{-\delta}<\ln ^{-\delta}(1+u)<(e-1)^{\delta} u^{-\delta}
$$

are true.

Using (47) and (50) we can check, that for all $y>0$ and $0<\alpha, \beta, \gamma<1 / 2$ the functions $\left(e^{-y|u|^{\alpha}}\right)_{u^{\prime}}^{\prime}\left(e^{-y \psi_{\beta}(|u|)}\right)_{u}^{\prime}$ and $\left(e^{-y \psi_{\gamma}(|u|)}\right)_{u}^{\prime}$ belong to the space $L_{2}$, and for $1 / 2 \leq \alpha, \beta, \gamma<1$ they do not belong to it. Hence, in the case $0<\alpha, \beta, \gamma<1 / 2$ the delta-like kernels $\Psi_{y}^{\alpha}(x), \Psi_{y, \beta}(x)$ and $\Psi_{y, \gamma}(x)$ satisfy the conditions of Theorem 3 .

In view of the fact that the necessary condition for absolute integrability of the Fourier transform $F(f)$ is a continuity and boundedness almost everywhere of the function $f$. Therefore, from the equalities (29) we get, that the necessary condition for the functions $x \Psi_{y}^{\alpha}(x)$, $x \Psi_{y, \beta}(x)$ and $x \Psi_{y, \gamma}(x)$ to be absolute integrable on the whole real axis, is that the functions $\left(e^{-y|u|^{\alpha}}\right)_{u^{\prime}}^{\prime}\left(e^{-y \psi_{\beta}(|u|)}\right)_{u}^{\prime}$ and $\left(e^{-y \psi_{\gamma}(|u|)}\right)_{u}^{\prime}$ are almost everywhere bounded for all fixed $y>0$. If $1 / 2 \leq \alpha, \beta, \gamma<1$, then the relations (47) yield that the functions $\left(e^{-y|u|^{\alpha}}\right)_{u}^{\prime},\left(e^{-y \psi_{\beta}(|u|)}\right)_{u}^{\prime}$ and $\left(e^{-y \psi_{\gamma}}(|u|)\right)_{u}^{\prime}$ are not bounded on the whole real axis. That is, for $1 / 2 \leq \alpha, \beta, \gamma<1$ the delta-like kernels $\Psi_{y}^{\alpha}(x), \Psi_{y, \beta}(x)$ and $\Psi_{y, \gamma}(x)$ do not satisfy the conditions of Theorem 3.

\section{References}

[1] Abdullayev F.G., Bushev D.M., Imashkyzy M., Kharkevych Yu.I. Isometry of the subspaces of solutions of systems of differential equations to the spaces of real functions. Ukrainian Math. J. 2020, 71 (8). 1153-1172. doi:10.1007/s11253-019-01705-9 (translation of Ukrain. Mat. Zh. 2019, 71 (8), 1011-1027. (in Ukrainian))

[2] Akhiezer N.I. Lectures on Approximation Theory. Nauka, Moskow, 1965. (in Russian)

[3] Bochner S. Lectures on Fourier Integrals. Fizmatgiz, Moskow, 1962. (in Russian)

[4] Bushev D.M. Isometry of functional spaces with diferent number of variables. Ukrainian Math. J. 1998,50 (8), 1170-1191. doi:10.1007/BF02513090 (translation of Ukrain. Mat. Zh. 1998, 50 (8), 1027-1045. (in Ukrainian))

[5] Bushev D.M., Kharkevych Yu.I. Finding solution subspaces of the Laplace and heat equations isometric to spaces of real functions and some of their applications. Math. Notes 2018, 103 (5-6), 869-880. doi: 10.1134/S0001434618050231

[6] Bushev D.M., Kharkevych Yu.I. Conditions of convergence almost everywhere for the convolution of a function with delta-shaped kernel to this function. Ukrainian Math. J. 2016, 67 (11) 1643-1661. doi:10.1007/s11253-016-1180-y (translation of Ukrain. Mat. Zh. 2015, 67 (11), 1461-1476. (in Ukrainian))

[7] Bushev D.N. Isometry of the functional spaces with different number of variables and some its applications in the theory of approximation of functions. J. Autom. Inf. Sci. 2019, 51 (1), 70-77. doi:10.1615/JAutomatInfScien.v51.i1.70 
[8] Bushev D.N. On some applications of isometry of functional spaces in applied mathematics. J. Autom. Inf. Sci. 2020, 52 (2), 53-62. doi:10.1615/JAutomatInfScien.v52.i2.50

[9] Hembars'ka S.B. On Boundary values of three-harmonic Poisson integral on the boundary of a unit disk. Ukrainian Math. J. 2018, 70 (7), 1012-1021. doi:10.1007/s11253-018-1548-2 (translation of Ukrain. Mat. Zh. 2018, 70 (7), 876-884. (in Ukrainian))

[10] Kal'chuk I.V., Kharkevych Yu.I. Complete asymptotics of the approximation of function from the Sobolev classes by the Poisson integrals. Acta Comment. Univ. Tartu. Math. 2018, 22 (1), 23-36. doi:10.12697/ ACUTM.2018.22.03

[11] Korneichuk N.P. Extremal Problems of Approximation Theory. Nauka, Moskow, 1976. (in Russian)

[12] Natanson I.P. Theory of Functions of a Real Variable. Nauka, Moskow, 1974. (in Russian)

[13] Nikol'skii S.M. Approximation of Functions of Several Variables and Imbedding Theorems. Nauka, Moskow, 1969. (in Russian)

[14] Samko S.G., Kilbas A.A., Marichev O.I. Integrals and Derivatives of Fractional Order and Some of Their Applications. Nauka i Tekhnika, Minsk, 1987. (in Russian)

Received 10.07.2021

Revised 09.12.2021

Бушев А.М., Абдулаєв Ф.Г., Кальчук І.В., Імашкизи М. Застосування ізометричності функиіональних просторів з різною кількістю змінних у теорії наближення функиій // Карпатські матем. публ. — 2021. - Т.13, №3. - С. 805-817.

У роботі знайдено інтегральні зображення для функціональних просторів, ізометричних просторам цілих функцій експоненціального типу, які необхідні для наведення прикладів рівності апроксимаційних характеристик у функціональних просторах, ізометричних просторам неперіодичних функцій. Отримано достатні умови для невід'ємності дельтаподібних ядер, які використовуються для побудови ізометричних функціональних просторів з різною кількістю змінних.

Ключові слова і фрази: дельтаподібне ялро, ізометричність, простір згорток, апроксимативна характеристика. 